\title{
Holographic charm and bottom pentaquarks. II. Open and hidden decay widths
}

\author{
Yizhuang Liu $\odot^{*}$ \\ Institute of Theoretical Physics, Jagiellonian University, 30-348 Kraków, Poland \\ Maciej A. Nowak ${ }^{\dagger}$ \\ Institute of Theoretical Physics and Mark Kac Center for Complex Systems Research, Jagiellonian University, \\ 30-348 Kraków, Poland \\ Ismail Zahed \\ Center for Nuclear Theory, Department of Physics and Astronomy, Stony Brook University, \\ Stony Brook, New York 11794-3800, USA
}

(Received 7 September 2021; accepted 31 October 2021; published 17 December 2021)

\begin{abstract}
We analyze the decay modes of the three $\left[\frac{1}{2} \frac{1}{2}\right]_{S=0,1}$ and $\left[\frac{1}{2} \frac{3}{2}\right]_{S=1}$ nonstrange pentaquarks with hidden charm and bottom, predicted by holographic QCD in the heavy quark limit. In leading order, the pentaquarks are degenerate and stable by heavy quark symmetry. At next to leading order, the spin interactions lift the degeneracy and cause the pentaquarks to decay. We show that the open charm (bottom) decay modes dwarf the hidden charm (bottom) ones, with total widths that are consistent with those recently reported by $\mathrm{LHCb}$ for charm pentaquarks. Predictions for bottom pentaquarks are given. The result of the current paper will be used in the part III of the current series of paper [Y. Liu, K. A. Mamo, M. A. Nowak, and I. Zahed, following paper, Holographic charm and bottom pentaquarks. III. Excitations through photo-production of heavy mesons, Phys. Rev. D 104, 114023 (2021).].
\end{abstract}

DOI: $10.1103 /$ PhysRevD.104.114022

\section{INTRODUCTION}

The LHCb high statistics analysis [1] shows that the previously reported $P_{c}^{+}(4450)$ [2] splits into two narrow peaks $P_{c}^{+}(4440)$ and $P_{c}^{+}(4457)$ just below the $\Sigma_{c}^{+} \bar{D}^{* 0}$ threshold, with the appearance of a new and narrow $P_{c}^{+}(4312)$ state right below the $\Sigma_{c}^{+} \bar{D}^{0}$. The evidence for the old and broad $P_{c}^{+}$(4380) [2] has now weakened. The reported charm pentaquark widths are narrow [1],

$$
\begin{array}{ll}
m_{P_{c}}=4311.9 \pm 0.7 \mathrm{MeV}, & \Gamma_{P_{c}}=9.8 \pm 2.7 \mathrm{MeV}, \\
m_{P_{c}}=4440.3 \pm 1.3 \mathrm{MeV}, & \Gamma_{P_{c}}=20.6 \pm 4.9 \mathrm{MeV}, \\
m_{P_{c}}=4457.3 \pm 0.6 \mathrm{MeV}, & \Gamma_{P_{c}}=6.4 \pm 2.0 \mathrm{MeV}
\end{array}
$$

The $P_{c}(4312)$ is observed to be $10 \mathrm{MeV}$ below the $\Sigma_{c}^{+} \bar{D}^{0}$ threshold, and the $P_{c}(4457)$ just $5 \mathrm{MeV}$ below the $\Sigma_{c}^{+} \bar{D}^{*}$ threshold illustrated in Fig. 1 from [1], a strong indication

\footnotetext{
*yizhuang.liu@uj.edu.pl maciej.a.nowak@uj.edu.pl

*ismail.zahed@stonybrook.edu
}

Published by the American Physical Society under the terms of the Creative Commons Attribution 4.0 International license. Further distribution of this work must maintain attribution to the author(s) and the published article's title, journal citation, and DOI. Funded by SCOAP ${ }^{3}$. of their molecular origin, as discussed by many (see [3-10] and references therein).

In the heavy quark limit, the heavy-light pair $\left[0^{-}, 1^{-}\right]=$ $\left[D, D^{*}\right]$ is degenerate and the $\Sigma_{c}^{+} \bar{D}^{0}$ and $\Sigma_{c}^{+} \bar{D}^{*}$ thresholds coalesce. As a result, the three reported pentaquark states become degenerate and stable by heavy quark symmetry. Three degenerate and stable pentaquark states with isospinspin-parity assignments $\left[\frac{1}{2} \frac{1}{2}^{-}\right]_{S=0,1}$ and $\left[\frac{1}{2} \frac{3}{2}\right]_{S=1}$, were predicted by holographic QCD, in the triple limit of a large number of colors, large 't Hooft gauge coupling $\lambda$ and a heavy quark mass $[11,12]$. The same assignments were subsequently made using the molecular construction [13-16].

The newly reported $P_{c}(4337)$ with a width of $29 \mathrm{MeV}$ at $3 \sigma$ significance [17] appears to overlap with the reported $P_{c}(4312)$ at $7 \sigma$ significance, and is not supported by our holographic analysis of the low-lying pentaquark states. The excited even and odd parity holographic pentaquark states $P_{c}^{*}$ lie higher in mass, and are likely much broader in phase space $[11,12,18]$.

Holographic pentaquarks are composed of heavy-light mesons bound to a topological instanton core in bulk. They are the dual of a nucleon core bound to heavy-light mesons at the boundary. In the heavy quark limit, the pentaquarks with hidden charm and bottom are degenerate, heavy and stable $[11,12,19,20]$. Away from the heavy quark limit, spinspin and spin-orbit forces lift the degeneracy and cause them to decay, as we will show below. This work is a follow-up on 




FIG. 1. LHCb measurements of the $P_{c}$ states fitted with three Breit-Wigner distributions (red solid curve and fitted background black solid curve), with the mass thresholds for the $\Sigma_{c}^{+} \bar{D}^{0}$ and $\Sigma_{c}^{+} \bar{D}^{* 0}$ final states from [1] shown for comparison.

our recent reanalysis of the charm and bottom pentaquark states including the spin effects, to which we refer for completeness [18].

The organization of the paper is as follows: In Sec. II we briefly review the essential aspects of the holographic construction in leading order in the heavy quark mass. In Sec. III, we detail the spin contributions to order $1 / m_{H}$ which are at the origin of the two-body decay of the pentaquarks with open charm final states. In Sec. IV we show how the two-body decay channel with hidden charm can be extracted from a Witten diagram in bulk. We derive a number of model independent ratios for the decay modes for both charm and bottom pentaquarks. For charm pentaquarks, they compare well to the total widths recently reported by LHCb. Our conclusions are in Sec. V. We include complementary appendixes for completeness.

\section{HOLOGRAPHIC HEAVY-LIGHT EFFECTIVE ACTION}

The D4-D8-D $\overline{8}$ setup for light flavor branes is standard [21]. The minimal modification that accommodates heavy mesons makes use of an extra-heavy brane, as discussed in $[11,22]$. The effective action consists of the non-Abelian Dirac-Born-Infeld (DBI), Chern-Simons (CS) and mass term

$$
\begin{aligned}
S_{\mathrm{DBI}} \approx & -\kappa \int d^{4} x d z \operatorname{Tr}\left(\mathbf{f}(z) \mathbf{F}_{\mu \nu} \mathbf{F}^{\mu \nu}+\mathbf{g}(z) \mathbf{F}_{\mu z} \mathbf{F}^{\nu z}\right) \\
& -\frac{1}{2} m_{H}^{2} \int d^{4} x d z \operatorname{Tr}\left(\Phi_{M}^{\dagger} \Phi_{M}\right) .
\end{aligned}
$$

The warping factors are

$$
\mathbf{f}(z)=\frac{R^{3}}{4 U_{z}}, \quad \mathbf{g}(z)=\frac{9}{8} \frac{U_{z}^{3}}{U_{K K}},
$$

with $U_{z}^{3}=U_{K K}^{3}+U_{K K} z^{2}$ and $\kappa \equiv a \lambda N_{c}$ and $a=1 /$ $\left(216 \pi^{3}\right)$ in units of $M_{K K}$ [21]. Our conventions are $(-1,1,1,1,1)$, with $A_{M}^{\dagger}=-A_{M}$ and the labels $M, N$ running over $\mu, z$ only in this section. The effective fields in the field strengths are $[11,22]$

$\mathbf{F}_{M N}=\left(\begin{array}{cc}F_{M N}-\Phi_{[M} \Phi_{N]}^{\dagger} & \partial_{[M} \Phi_{N]}+A_{[M} \Phi_{N]} \\ -\partial_{[M} \Phi_{N]}^{\dagger}-\Phi_{[M}^{\dagger} A_{N]} & -\Phi_{[M}^{\dagger} \Phi_{N]}\end{array}\right)$.

The matrix valued 1-form gauge field is

$$
\mathbf{A}=\left(\begin{array}{cc}
A & \Phi \\
-\Phi^{\dagger} & 0
\end{array}\right)
$$

For $N_{f}=2$, the naive Chern-Simons 5-form is

$$
S_{\mathrm{CS}}=\frac{i N_{c}}{24 \pi^{2}} \int_{M_{5}} \operatorname{Tr}\left(A F^{2}-\frac{1}{2} A^{3} F+\frac{1}{10} A^{5}\right) .
$$

For $N_{f}$ coincident branes, the $\Phi$ multiplet is massless, but for separated branes they are massive with $m_{H}$ fixed by the separation between the heavy and light branes. We follow [22] and fix it by the heavy meson masses $M_{D}=1870 \mathrm{MeV}$ (charmed) and $M_{B}=5279 \mathrm{MeV}$ (bottomed) using

$$
M_{D, B}=m_{H}+\frac{M_{K K}}{2 \sqrt{2}} .
$$

While the use of one heavy brane is well suited for single heavy baryons, doubly heavy baryons require a priori two heavy branes. However, in the heavy quark limit and to order $1 / m_{H}$, including the spin-orbit effects as we will discuss below, there is no need to double the heavy branes. Such doubling will be needed if e.g., spin-spin interactions are retained to order $1 / m_{H}^{2}$.

In the coincident brane limit, light baryons are interchangeably described as a flavor instanton or a D4 brane wrapping the $S^{4}$. The instanton size is small, with $\rho \sim 1 / \sqrt{\lambda}$ after balancing the order $\lambda$ bulk gravitational attraction with the subleading and of order $\lambda^{0} \mathrm{U}(1)$ induced topological repulsion [21]. The $\mathrm{O}(4)$ instanton gauge field is of the form

$$
A_{M}(y)=-\bar{\sigma}_{M N} \partial_{N} F(y),\left.\quad F_{z m}(y)\right|_{|y|=R}=0 .
$$

Since $\rho \sim 1 / \sqrt{\lambda}$ is the typical instanton size, it is convenient to rescale the fields

$$
\begin{aligned}
\left(x_{0}, x_{M}\right) & \rightarrow\left(x_{0}, x_{M} / \sqrt{\lambda}\right), \\
\sqrt{\lambda} \rho \rightarrow \rho,\left(A_{0}, A_{M}\right) & \rightarrow\left(A_{0}, \sqrt{\lambda} A_{M}\right)
\end{aligned}
$$

with the leading order equations of motion 
$D_{M} F_{M N}=0, \quad \partial_{M}^{2} A_{0}=-\frac{1}{32 \pi^{2} a} F_{a M N} \star F_{a M N}$.

Similarly, the bound heavy-light fields $\left(\Phi_{0}, \Phi_{M}\right)$ are rescaled using

$$
\left(\Phi_{0}, \Phi_{M}\right) \rightarrow\left(\Phi_{0}, \sqrt{\lambda} \Phi_{M}\right) .
$$

Following the rescaling, the effective action for the light gauge fields $\left(A_{0}, A_{M}\right)$ and the heavy fields $\left(\Phi_{0}, \Phi_{M}\right)$ in leading order is $[11,22]$

$$
\mathcal{L}=a N_{c} \lambda \mathcal{L}_{0}+a N_{c} \mathcal{L}_{1}+\mathcal{L}_{\mathrm{CS}}
$$

with

$$
\mathcal{L}=a N_{c} \lambda \mathcal{L}_{0}+a N_{c}\left(\mathcal{L}_{1}+\tilde{\mathcal{L}}_{1}\right)+\mathcal{L}_{\mathrm{CS}}
$$

with each contribution given by

$$
\begin{aligned}
\mathcal{L}_{0}= & -\left(D_{M} \Phi_{N}^{\dagger}-D_{N} \Phi_{M}^{\dagger}\right)\left(D_{M} \Phi_{N}-D_{N} \Phi_{M}\right)+2 \Phi_{M}^{\dagger} F_{M N} \Phi_{N}, \\
\mathcal{L}_{1}= & +2\left(D_{0} \Phi_{M}^{\dagger}-D_{M} \Phi_{0}^{\dagger}\right)\left(D_{0} \Phi_{M}-D_{M} \Phi_{0}\right)-2 \Phi_{0}^{\dagger} F^{0 M} \Phi_{M}-2 \Phi_{M}^{\dagger} F^{M 0} \Phi_{0}-2 m_{H}^{2} \Phi_{M}^{\dagger} \Phi_{M}, \\
\tilde{\mathcal{L}}_{1}= & +\frac{z^{2}}{3}\left(D_{i} \Phi_{j}-D_{j} \Phi_{i}\right)^{\dagger}\left(D_{i} \Phi_{j}-D_{j} \Phi_{i}\right)-2 z^{2}\left(D_{i} \Phi_{z}-D_{z} \Phi_{i}\right)^{\dagger}\left(D_{i} \Phi_{z}-D_{z} \Phi_{i}\right) \\
& -\frac{2}{3} z^{2} \Phi_{i}^{\dagger} F_{i j} \Phi_{j}+2 z^{2}\left(\Phi_{z}^{\dagger} F_{z i} \Phi_{i}+\text { c.c. }\right), \\
& \mathcal{L}_{\mathrm{CS}}=-\frac{i N_{c}}{16 \pi^{2}} \Phi^{\dagger}\left(d A+A^{2}\right) D \Phi-\frac{i N_{c}}{16 \pi^{2}}(D \Phi)^{\dagger}\left(d A+A^{2}\right) \Phi+\mathcal{O}\left(\Phi^{3}\right) .
\end{aligned}
$$

The expansion around the heavy quark limit will be sought using $\Phi_{M}=\phi_{M} e^{-i m_{H} x_{0}}$ for particles and $m_{H} \rightarrow-m_{H}$ for antiparticles. In particular, we have in leading order $[11,12]$

$\mathcal{L}_{0}=-\frac{1}{2}\left|f_{M N}-\star f_{M N}\right|^{2}+2 \phi_{M}^{\dagger}\left(F_{M N}-\star F_{M N}\right) \phi_{N}$

subject to the constraint equation $D_{M} \phi_{M}=0$ with $f_{M N}=\partial_{[M} \phi_{N]}+A_{[M} \phi_{N]}$, and

$$
\begin{aligned}
\frac{\mathcal{L}_{1}}{a N_{c}} & \rightarrow 4 m_{H} \phi_{M}^{\dagger} i D_{0} \phi_{M}, \\
\mathcal{L}_{\mathrm{CS}} & \rightarrow \frac{m_{H} N_{c}}{16 \pi^{2}} \phi_{M}^{\dagger} \star F_{M N} \phi_{N} .
\end{aligned}
$$

For self-dual fields $F_{M N}=\star F_{M N}$, and the minimum of Eq. (15) is reached for $f_{M N}=\star f_{M N}$. As a result, the combination $\psi=\bar{\sigma}_{M} \phi_{M}$ with $\sigma_{M}=(i, \vec{\sigma})$ obeys the zero mode equation $\sigma_{M} D_{M} \psi=D \psi=0$. While binding to the core instanton, the heavy mesons with spin 1 transmute to a Weyl fermion with spin $\frac{1}{2}[11,12]$.

The holographic charmed pentaquark states are ultimately bound topological molecules with hidden charm, without the ambiguities related to the type of meson exchange to use and the details of the form factors (hard core), a challenge for most molecular constructions (see [3-10,23,24] and references therein). The dual of the hard core is the instanton core which is universal and fixed by gauge-gravity interactions in bulk. The dual of the meson exchanges are bulk light and heavy gauge fields regulated by unique D-brane gauge interactions in conformity with chiral symmetry, vector dominance and heavy quark symmetry at the boundary. We now address their strong decay modes using the effective action (13).

\section{OPEN CHARM DECAYS}

The charmed pentaquark states decay modes can proceed through either open charm channels, given their proximity to the $\Sigma_{c}\left[\bar{D}, \bar{D}^{*}\right]$ thresholds [1], or hidden charm channels such as $J / \Psi$ as originally observed [2]. For clarity, all the analyses to follow will be carried with the decay kinematics using $P_{c}(4440)$. The final results will be tabulated for all three charm pentaquark states recently reported, and extended to the yet to be observed bottom pentaquarks.

The decay modes follow from the coupling between the background classical field $\Phi_{M}$ sourced by the baryonic moduli, and the fluctuating heavy-light meson field $\delta \Phi_{M}$ [22]. Note that our classical field configuration $\left(\Phi_{0}, \Phi_{M}\right)$ only solves the equation of motion to leading order in $1 / \lambda$. Therefore under the shift $\Phi_{M} \rightarrow \delta \Phi_{M}$ there are linear terms in $\delta \Phi_{M}$. They do not affect the stability of the instanton core.

More specifically, the linear contributions in leading order in $m_{H}$ are

$$
\begin{aligned}
\delta \mathcal{L}= & 4 \operatorname{iaN}_{c} m_{H}\left(\delta \Phi_{M}^{\dagger} \hat{A}_{0} \Phi_{M}+\Phi_{M}^{\dagger} \hat{A}_{0} \delta \Phi_{M}\right) \\
& +\frac{N_{c} m_{H}}{8 \pi^{2}}\left(\delta \Phi_{M}^{\dagger} F_{M N} \Phi_{N}+\Phi_{M}^{\dagger} F_{M N} \delta \Phi_{N}\right) .
\end{aligned}
$$

The first contribution is kinetic and the second contribution is topological (Chern-Simons term). For vector mesons we have 
$\delta \Phi_{M}(t, \vec{x}, z)=\epsilon_{M} e^{-i M_{n} t} \phi_{n}(z), \quad \delta \Phi_{z}(t, \vec{x}, z)=0$,

with the interaction term

$$
\begin{aligned}
\delta L= & \frac{i m_{H} N_{c}}{2 \pi^{2} \sqrt{16 m_{H} a N_{c}}} \frac{c \phi_{n}(Z)}{\left(X^{2}+\rho^{2}\right)^{\frac{5}{2}}} \\
& \times\left(1+\frac{5 \rho^{2}}{2\left(X^{2}+\rho^{2}\right)}\right)\left(\vec{\epsilon} \dagger \cdot \vec{\sigma} \chi_{Q}-\chi_{Q}^{\dagger} \vec{\sigma} \cdot \vec{\epsilon}\right) .
\end{aligned}
$$

The heavy-light mesonic wave functions in bulk satisfy $\left(\tilde{Z}=\sqrt{m_{H}} Z\right)$

$$
-\frac{d^{2} \phi_{n}(\tilde{Z})}{d \tilde{Z}^{2}}+\frac{\tilde{Z}^{2}}{2} \phi_{n}(\tilde{Z})=\left(m_{n}^{2}-m_{H}^{2}\right) \phi_{n}(\tilde{Z}),
$$

with the normalized solutions [22]

$\phi_{n}(\tilde{Z})=\frac{1}{\sqrt{2 \tilde{\kappa}}} \frac{1}{\sqrt{2^{n} n !}}\left(\frac{\sqrt{2}}{2 \pi} m_{H}\right)^{\frac{1}{4}} e^{-\frac{\sqrt{2} \tilde{Z}^{2}}{4}} H_{n}\left[\left(\frac{\sqrt{2}}{2}\right)^{\frac{1}{2}} \tilde{Z}\right]$

and the Reggeized mass spectrum

$$
m_{n}^{2} \approx m_{H}^{2}+\frac{7 m_{H} m_{\rho}}{4}\left(n+\frac{1}{2}\right)
$$

Note that the two brane tensions $\tilde{\kappa}$ in the heavy-light sector and $\kappa$ in the light-light sector are identified, for bulk filling branes. However, here, we will keep them separate phenomenologically, as we discuss below. With this in mind, the Hamiltonian following from Eq. (19) after integration over $d Z d^{3} X$ reads

$$
\delta H=i \alpha \epsilon_{i}^{\star} \tau_{i} \lambda-i \alpha \lambda^{\dagger} \tau_{i} \epsilon_{i}+\alpha \epsilon^{\dagger} \lambda+\alpha \lambda^{\dagger} \epsilon
$$

with the moduli coefficient

$$
\alpha\left(\rho, \frac{Z}{\rho}\right)=\frac{\sqrt{2} \rho N_{c}}{3 \pi^{2} \sqrt{a N_{c}}\left(Z^{2}+\rho^{2}\right)} \int d Z \phi_{n}(Z),
$$

which depend on the specifics of the moduli wave functions which are detailed in Appendix A.

\section{A. Generic form of the spin interaction}

If we fix the vector meson polarization to say $\epsilon_{M}$, then the sole coupling to the angular momentum is rotorlike $\chi^{a} F^{a}(\chi)$ which contributes to the Hamiltonian as $\vec{L} \cdot \vec{F}$. It conserves angular momentum $l$ and cannot cause an angular momentum transition necessary for the open channel decays. However, a close inspection shows that we need to consider the mismatch caused by the gauge transformation $V$ that acts solely on the instanton profile but not on the external field [25]. Including this gauge transformation amounts to the substitution

$$
\delta \Phi_{M} \rightarrow V^{-1}(t, z, x) \delta \Phi_{M} \approx\left(a_{4}-i \vec{a} \cdot \vec{\tau}\right) \delta \Phi_{M} .
$$

As a result, the change in the Lagrangian can still be obtained from Eq. (19) with the replacement

$$
\epsilon_{M} \rightarrow\left(a_{4}-i \vec{a} \cdot \vec{\tau}\right) \epsilon_{M},
$$

which allows for the transition from $l$ to $l \pm 1$. We conclude that by expanding in linear order in $\delta \Phi_{M}$, we can generate a transition vertex with net angular momentum change by 1 . Therefore the transitions from $P_{c}$ with $l=1$ to $\Lambda_{c}$ with $l=0$ and to $\Sigma_{c}$ with $l=2$ are all possible.

\section{B. General transition vertices}

The vertex responsible for the decay to a vector meson $P_{c} \rightarrow D^{\star} p$ follows from

$\delta H=i \alpha \epsilon_{i}^{\star}\left(a_{4}+i \vec{a} \cdot \tau\right) \tau_{i} \lambda-i \alpha \lambda^{\dagger} \tau_{i}\left(a_{4}-i \vec{a} \cdot \tau\right) \epsilon_{i}$,

or more specifically the matrix element

$$
\left\langle l^{\prime} m^{\prime} ; \frac{1}{2} s|\delta H| l m_{1} ; S m_{2}\right\rangle \text {. }
$$

(1) For $S=0$, the transition matrix for $P_{c} \rightarrow D+p$ with a scalar meson final state is

$$
\delta H=\alpha \epsilon^{\dagger}\left(a_{4}+i \vec{a} \cdot \vec{\tau}\right) \lambda+\alpha \lambda^{\dagger}\left(a_{4}-i \vec{a} \cdot \vec{\tau}\right) \epsilon,
$$

and the corresponding transition amplitude is

$$
\begin{aligned}
& \mathcal{M}\left(P_{c}, S=0 \rightarrow D(\epsilon)+l^{\prime} m^{\prime}+\lambda_{s}\right) \\
& =\alpha \epsilon^{\dagger}\left\langle l^{\prime} m^{\prime}\left|a_{4}+i \vec{a} \cdot \vec{\tau}\right| l m\right\rangle \sigma_{2} \lambda_{s} .
\end{aligned}
$$

The transition amplitude with a vector meson final state is

$$
\begin{aligned}
\mathcal{M} & \left(P_{c}, S=0 \rightarrow D^{\star}(\vec{\epsilon})+l^{\prime} m^{\prime}+\lambda_{s}\right) \\
= & \alpha\left\langle l^{\prime} m^{\prime}\left|i a_{4}\right| \operatorname{lm}\right\rangle \vec{\epsilon}^{\star} \cdot \vec{\tau} \sigma_{2} \lambda_{s}^{\star} \\
& -\alpha\left\langle l^{\prime} m^{\prime}\left|i a_{i}\right| l m\right\rangle\left(\vec{\epsilon}^{\star} \times \vec{\tau}\right)_{i} \sigma_{2} \lambda_{s}^{\star} \\
& -\alpha\left\langle l^{\prime} m^{\prime}\left|i a_{i}\right| l m\right\rangle \epsilon_{i}^{\star} \sigma_{2} \lambda_{s} .
\end{aligned}
$$

(2) For $S=1$, the transition amplitude for $P_{c} \rightarrow D+p$ with a scalar meson final state is

$$
\begin{aligned}
& \mathcal{M}\left(P_{c}, 1 S \rightarrow D(\epsilon)+l^{\prime} m^{\prime}+\lambda_{s}\right) \\
& =\alpha\left(\epsilon^{\dagger}\left\langle l^{\prime} m^{\prime}\left|a_{4}+i \vec{a} \cdot \vec{\tau}\right| l m\right\rangle\right)_{s^{\prime}} C_{s^{\prime} s}^{1 ; S} \lambda_{s}
\end{aligned}
$$

after replacing $\sigma_{2}$ by the general Clebsch-Gordan coefficient for $\frac{1}{2}+\frac{1}{2}=1$, 


$$
\sigma_{2} \rightarrow C_{s s^{\prime}}^{1 ; s+s^{\prime}}=\delta_{s s^{\prime}}+\sigma_{s s^{\prime}}^{1}
$$

The corresponding transition amplitude with a vector meson final state is

$$
\begin{aligned}
\mathcal{M} & \left(P_{c}, 1 S \rightarrow D^{\star}(\vec{\epsilon})+l^{\prime} m^{\prime}+\lambda_{s}\right) \\
= & \alpha\left\langle l^{\prime} m^{\prime}\left|i a_{4}\right| l m\right\rangle\left(\vec{\epsilon}^{\star} \cdot \vec{\tau}\right)_{s^{\prime}} C_{s^{\prime} s}^{1 ; S} \lambda_{s}^{\star} \\
& -\alpha\left\langle l^{\prime} m^{\prime}\left|i a_{i}\right| l m\right\rangle\left(\vec{\epsilon}^{\star} \times \vec{\tau}\right)_{i s^{\prime}} C_{s^{\prime} s}^{1 ;} \lambda_{s}^{\star} \\
& -\alpha\left\langle l^{\prime} m^{\prime}\left|i a_{i}\right| l m\right\rangle \epsilon_{i s^{\prime}}^{\star} C_{s^{\prime} s}^{1 ;} \lambda_{s}^{\star} .
\end{aligned}
$$

In a typical decay, we need to combine $\lambda_{s}$ with $l^{\prime} m^{\prime}$ to form the finite $J$ final state, and combine $S$ with $m$ to form the finite $J$ initial state. Then we need to square and sum over spin. We now apply this to a number of decay channels with open charm.

$$
\text { C. } \boldsymbol{P}_{\boldsymbol{c}} \rightarrow \boldsymbol{\Lambda}_{c}+\bar{D} \text { decay }
$$

The pentaquark decay through $\Lambda_{c}$ is larger than through $\Sigma_{c}$ or $\Sigma_{c}^{*}$, given the larger access to phase space. A quick inspection of quantum numbers show that the decay process

$$
\left[P_{c}(4440)\left[\frac{1}{2} \frac{1^{-}}{2}\right]_{0}\right] \rightarrow\left[\Lambda_{c}(2286) 0 \frac{1}{2}^{+}\right]+\left[\bar{D}(1870) \frac{1}{2} 0^{-}\right]
$$

is quadrupolar with $l=2$, since the $l=0$ is forbidden by momentum conservation and $l=1$ by parity. The final meson decay momentum $|\vec{p}| \approx 778 \mathrm{MeV}$, so the decay produced is nonrelativistic.

$$
\text { 1. } S=0
$$

For $S=0$, we need

$$
\begin{aligned}
& \mathcal{M}\left(P_{c}, S=0 \rightarrow D(\epsilon)+\Lambda_{c}(s)\right) \\
& =\alpha \epsilon^{\dagger}\left\langle 0\left|a_{4}+i \vec{a} \cdot \vec{\tau}\right| \beta \dot{\beta}\right\rangle \frac{1}{\sqrt{2}} \sigma_{2} \lambda_{s} .
\end{aligned}
$$

After summing over spin we only need to consider

$$
\frac{1}{2} \sum_{\beta, \dot{\beta}} \alpha^{2} \operatorname{tr}\left\langle 00\left|a_{4}+i \vec{a} \cdot \vec{\tau}\right| \beta \dot{\beta}\right\rangle\left\langle\beta \dot{\beta}\left|a_{4}-i \vec{a} \cdot \vec{\tau}\right| 00\right\rangle .
$$

The hyperspherical harmonics $|\dot{\beta}\rangle$ can be represented in terms of $2 \times 2$ matrices as

$$
\Psi_{\alpha \dot{\alpha}}^{l=1}(a)=\frac{\sqrt{2}}{\sqrt{\Omega_{4}}}(\sigma \cdot a)_{\alpha \dot{\alpha}},
$$

where $\Omega_{4}=2 \pi^{2}$. We now observe that

$$
\left\langle 0\left|(\bar{\sigma} \cdot a)_{\dot{\alpha}, \alpha}\right|(\sigma \cdot a)^{\dot{\beta} \dot{\beta}}\right\rangle=\frac{\sqrt{2}}{2} \frac{1}{\sqrt{2}} \delta_{\alpha}^{\beta} \frac{1}{\sqrt{2}} \delta_{\dot{\alpha}}^{\dot{\beta}}
$$

In terms of these, one has

$$
\sum_{\beta \dot{\beta}} \alpha^{2} \operatorname{tr}\left\langle 00\left|a_{4}+i \vec{a} \cdot \vec{\tau}\right| \beta \dot{\beta}\right\rangle\left\langle\beta \dot{\beta}\left|a_{4}-i \vec{a} \cdot \vec{\tau}\right| 00\right\rangle=\frac{1}{2} \alpha^{2},
$$

so that

$$
\Gamma_{P_{c}, S=0 \rightarrow D(\epsilon)+\Lambda_{c}(s)}=\frac{|\vec{p}|}{2 \pi} \frac{m_{H}^{\frac{1}{2}} M_{K K}^{\frac{1}{2}}}{2 \tilde{\kappa}} \frac{1}{4}\langle\alpha\rangle^{2} \times \frac{1}{4} \equiv \Gamma,
$$

where $\frac{1}{4}$ comes from the initial state averaging over spin and isospin, and

$$
\langle\alpha\rangle_{l=1 \rightarrow l=0}=4.08 / \pi .
$$

The decay width (40) is fixed by kinematics $|\vec{p}| \sim 778 \mathrm{MeV}$ and the three holographic parameters $\tilde{\kappa}, M_{K K}, m_{H}$.

In general, the brane tension for the heavy-light fields $\tilde{\kappa}$ and that of the light-light fields $\kappa$ are the same. Here we choose to treat them separately. In [18] the three parameters $\kappa, M_{K K}, m_{H}$ were fixed to reproduce globally the charm and bottom baryons as well as the pentaquarks with hidden charm and bottom. Here, their adjustment to the three observed masses will be subsumed. The additional $\tilde{\kappa}$ parameter will be fixed by one measured width as we detail below. All other partial and total widths will follow in units of $\Gamma$ as predictions, for both charm and bottom.

\section{2. $S=1$}

For $S=1$ we need

$$
\begin{aligned}
& \sum_{S, m, m^{\prime}} C_{\beta, S}^{J ; \beta+S}\left(C_{\beta^{\prime}, S}^{J ; \beta^{\prime}+S}\right)^{\dagger}\langle\alpha\rangle^{2} \operatorname{tr}\langle 00| a_{4} \\
& +i \vec{a} \cdot \vec{\tau}|\beta \dot{\beta}\rangle C^{1 S} C^{1 S \dagger}\left\langle\dot{\beta} \beta^{\prime}\left|a_{4}-i \vec{a} \cdot \vec{\tau}\right| 00\right\rangle \\
& =\sum_{s_{1}, s_{2}}\left|\left\langle S, s_{1}+s_{2} \mid \frac{1}{2}, s_{1} ; \frac{1}{2}, s_{2}\right\rangle\right|^{2}\left|\left\langle J, s_{1} \mid \frac{1}{2},-s_{2} ; S, s_{1}+s_{2}\right\rangle\right|^{2},
\end{aligned}
$$

where we also use $\left\langle 1, s_{1}+s_{2} \mid \frac{1}{2}, s_{1} ; \frac{1}{2}, s_{2}\right\rangle$ to denote the expansion coefficient of two spin $\frac{1}{2}$ to one spin 1 . In sum, the decay rate in which all the spins are summed over are the same, and is given by 


$$
\Gamma_{P_{c}(J, S) \rightarrow D(\epsilon)+\Lambda_{c}(s)}=\frac{|\vec{p}|}{2 \pi} \frac{m_{H}^{\frac{1}{2}} M_{K K}^{\frac{1}{2}}}{2 \tilde{\kappa}} \frac{1}{4}\langle\alpha\rangle^{2} \frac{1}{2(2 J+1)} \sum_{s_{1}, s_{2}}\left|\left\langle S, s_{1}+s_{2} \mid \frac{1}{2}, s_{1} ; \frac{1}{2}, s_{2}\right\rangle\right|^{2}\left|\left\langle J, s_{1} \mid \frac{1}{2},-s_{2} ; S, s_{1}+s_{2}\right\rangle\right|^{2} .
$$

We define

$$
f(J, S)=\sum_{s_{1}, s_{2}}\left|\left\langle S, s_{1}+s_{2} \mid \frac{1}{2}, s_{1} ; \frac{1}{2}, s_{2}\right\rangle\right|^{2}\left|\left\langle J, s_{1} \mid \frac{1}{2},-s_{2} ; S, s_{1}+s_{2}\right\rangle\right|^{2}
$$

with $f\left(\frac{1}{2}, 0\right)=1$ for $S=0$. For $S=1$ we have

$$
\begin{aligned}
f\left(J=S \pm \frac{1}{2}, S=1\right)= & \sum_{s_{1}, s_{2}= \pm \frac{1}{2}} \frac{1}{4}\left(1+\left|s_{1}+s_{2}\right|\right) \\
& \times\left(1 \mp \frac{4}{3} s_{1} s_{2}\right)=\frac{3}{2} \mp \frac{1}{6},
\end{aligned}
$$

hence the model independent ratios

$$
\frac{\Gamma_{P_{c}(J, S) \rightarrow D(\epsilon)+\Lambda_{c}(s)}}{\Gamma_{P_{c}\left(J^{\prime}, S^{\prime}\right) \rightarrow D(\epsilon)+\Lambda_{c}(s)}}=\frac{\left(2 J^{\prime}+1\right) f(J, S)}{(2 J+1) f\left(J^{\prime}, S^{\prime}\right)}
$$

or more explicitly

$$
\begin{aligned}
& \Gamma\left(S=0, J=\frac{1}{2}\right): \Gamma\left(S=1, J=\frac{1}{2}\right): \Gamma\left(S=1, J=\frac{3}{2}\right) \\
& =\frac{1}{2}: \frac{5}{6}: \frac{1}{3}
\end{aligned}
$$

whenever the decay mode is allowed kinematically.

\section{D. $\boldsymbol{P}_{\boldsymbol{c}} \rightarrow \boldsymbol{\Lambda}_{\boldsymbol{c}}+\overline{\boldsymbol{D}}^{*}$ decay}

The following decay width can be deduced from that of the scalar meson from the requirement of heavy quark symmetry:

$$
\left[P_{c}(4440)\left[\frac{1}{2} \frac{1-}{2}\right]_{0}\right] \rightarrow\left[\Lambda_{c}(2286) 0 \frac{1}{2}^{+}\right]+\left[\bar{D}^{*}(2010) \frac{1}{2} 1^{-}\right] .
$$

Indeed, the minimal Lagrangian reads in the case of $J=1 / 2$

$$
\bar{\psi}_{P_{c}}\left(D+\gamma^{5} \gamma^{\mu} D_{\mu}\right) \frac{1+\gamma \cdot v}{2} \psi_{\Lambda_{c}}+\text { c.c. }
$$

From these the ratio of the decay width for the scalar and the vector are proportional to

$$
\operatorname{tr} 1: \operatorname{tr} \sigma_{i} \sigma_{j} \epsilon_{i} \epsilon_{j}=1: 3
$$

so that

$$
\frac{\Gamma_{P_{c} \rightarrow \Lambda_{c}+\bar{D}^{*}}}{\Gamma_{P_{c} \rightarrow \Lambda_{c}+\bar{D}}}=3
$$

\section{E. $\boldsymbol{P}_{c} \rightarrow \boldsymbol{\Sigma}_{c}+\overline{\boldsymbol{D}}$ decay}

The spin-parity assignment of $\Sigma_{c}$ is that of $\Lambda_{c}$, so this decay mode is similar to the one we addressed earlier,

$$
\left[P_{c}(4440)\left[\frac{1}{2} \frac{1-}{2}\right]_{0}\right] \rightarrow\left[\Sigma_{c}(2453) 1 \frac{1}{2}^{+}\right]+\left[\bar{D}(1870) \frac{1}{2} 0^{-}\right],
$$

which is also quadrupolar with $l=2$. However, the width is expected to be smaller due to the narrower phase space. The final momentum is $|\vec{p}| \approx 502 \mathrm{MeV}$, so again the final kinematics is nonrelativistic. To carry the rate, we need the amplitude

$$
\begin{aligned}
& \mathcal{M}\left(P_{c}, S=0 \rightarrow D(\epsilon)+\Sigma_{c}(S)\right) \\
& =\alpha \epsilon^{\dagger}\left\langle 1 m_{1} ; 1 m_{2}\left|a_{4}+i \vec{a} \cdot \vec{\tau}\right| \beta \dot{\beta}\right\rangle \sigma_{2} \lambda_{s} C_{m_{1} s}^{\frac{1}{2} S},
\end{aligned}
$$

with the corresponding squared sum

$$
\begin{aligned}
& \sum_{\beta, \dot{\beta}, m_{1}, m_{1}^{\prime}, m_{2}, S} \operatorname{Tr}\left[\sigma_{2}\left\langle\beta \dot{\beta}\left|a_{4}-i \vec{a} \cdot \vec{\tau}\right| 1 m_{1} ; 1 m_{2}\right\rangle\right. \\
& \left.\times\left(C_{m_{1}}^{\frac{1}{2} S}\right)^{\dagger} C_{m_{1}^{\prime}}^{\frac{1}{2} S}\left\langle 1 m_{1}^{\prime} ; 1 m_{2}\left|a_{4}+i \vec{a} \cdot \vec{\tau}\right| \beta \dot{\beta}\right\rangle \sigma_{2}\right] .
\end{aligned}
$$

For the $S=1$ state we need the resummation 


$$
\begin{aligned}
& \sum_{\beta, \beta^{\prime}, \dot{\beta}, m_{1}, m_{1}^{\prime}, m_{2}, S, S^{\prime}} C_{\beta, S}^{J ; \beta+S}\left(C_{\beta, S}^{J ; \beta^{\prime}+S}\right)^{\dagger} \\
& \times \operatorname{Tr}\left[C^{1 S^{\dagger}}\left\langle\beta \dot{\beta}\left|a_{4}-i \vec{a} \cdot \vec{\tau}\right| 1 m_{1} ; 1 m_{2}\right\rangle\left(C_{m_{1}}^{\frac{1}{2} S^{\prime}}\right)^{\dagger} C_{m_{1}^{\frac{1}{2} S^{\prime}}}\right. \\
& \left.\times\left\langle 1 m_{1}^{\prime} ; 1 m_{2}\left|a_{4}+i \vec{a} \cdot \vec{\tau}\right| \beta^{\prime} \dot{\beta}\right\rangle C^{1 S}\right] .
\end{aligned}
$$

This can be achieved using the following identity in terms of Clebsch-Gordan coefficients:

$$
\begin{aligned}
& \left\langle 1, m_{1} ; 1, m_{2}\left|(\bar{\sigma} \cdot a)_{\dot{\alpha} \alpha}\right|(\sigma \cdot a)^{\beta \dot{\beta}}\right\rangle \\
& =\mathbb{A}\left\langle 1, m_{1} \mid \frac{1}{2}, \alpha ; \frac{1}{2},-\beta\right\rangle\left\langle 1, m_{2} \mid \frac{1}{2}, \dot{\alpha} ; \frac{1}{2},-\dot{\beta}\right\rangle,
\end{aligned}
$$

where $\mathbb{A}$ is a numerical number independent of spin. The minus sign follows from lowering $\beta$ and $\dot{\beta}$ down using $\sigma_{2}$ to form the spin sum. To evaluate $\mathbb{A}$, we may choose $\alpha=\dot{\alpha}=+$, and $\beta=\dot{\beta}=-$, then sum over $m_{1}$ and $m_{2}$, to obtain

$$
\left.|A|\right|^{2}=\frac{2}{\Omega_{4}} \sum_{P_{2}}\left(\int d \Omega_{4}\left(a_{1}^{2}+a_{2}^{2}\right) \Phi_{P_{2}}(a)\right)^{2}=\frac{1}{6},
$$

where the sum over $P_{2}$ ranges over all the nine independent hyperspherical harmonic functions for $l=2$.

Using the above results, the decay rate can be obtained by summing over all the spins,

$$
\begin{aligned}
\Gamma_{P_{c}(J, S) \rightarrow \Sigma_{c}+D}= & \frac{|\vec{p}| m_{H}^{\frac{1}{2}} M_{K K}^{\frac{1}{2}}}{4 \pi \tilde{\kappa}} \frac{\langle\alpha\rangle^{2}}{6(2 J+1)}|\mathbb{A}|^{2} \times \sum_{s_{1}, s_{2}}\left|\left\langle S, s_{1}+s_{2} \mid \frac{1}{2}, s_{1} ; \frac{1}{2}, s_{2}\right\rangle\right|^{2}\left|\left\langle J, s_{1} \mid \frac{1}{2},-s_{2} ; S, s_{1}+s_{2}\right\rangle\right|^{2} \\
& \times\left|\left\langle 1,0 \mid \frac{1}{2}, s_{1} ; \frac{1}{2},-s_{1}\right\rangle\right|^{2}\left|\left\langle\frac{1}{2},-s_{1} \mid 1,0 ; \frac{1}{2},-s_{1}\right\rangle\right|^{2} \\
& =\frac{|\vec{p}| m_{H}^{\frac{1}{2}} M_{K K}^{\frac{1}{2}}}{4 \pi \tilde{\kappa}} \frac{\langle\alpha\rangle^{2}}{36(2 J+1)}|\mathbb{A}|^{2} \times \sum_{s_{1}, s_{2}}\left|\left\langle S, s_{1}+s_{2} \mid \frac{1}{2}, s_{1} ; \frac{1}{2}, s_{2}\right\rangle\right|^{2}\left|\left\langle J, s_{1} \mid \frac{1}{2},-s_{2} ; S, s_{1}+s_{2}\right\rangle\right|^{2},
\end{aligned}
$$

where the additional factor of $\frac{1}{6}$ originates from

$$
\begin{aligned}
\left|\left\langle 1,0 \mid \frac{1}{2}, s_{1} ; \frac{1}{2},-s_{1}\right\rangle\right| & =\frac{1}{\sqrt{2}}, \\
\left|\left\langle\frac{1}{2},-s_{1} \mid 1,0 ; \frac{1}{2},-s_{1}\right\rangle\right| & =\sqrt{\frac{2}{3}} \times \frac{1}{\sqrt{2}} .
\end{aligned}
$$

This decay rate relates to the one for $\Lambda_{c}$, and the model independent ratio is

$$
\frac{\Gamma_{P_{c}(J, S) \rightarrow \Sigma_{c}+D}}{\Gamma_{P_{c}(J, S) \rightarrow \Lambda_{c}+D}}=\frac{502}{778} \times \frac{8}{36} \times \frac{4.97^{2}}{4.08^{2}}=0.574 .
$$

\section{F. $\boldsymbol{P}_{\boldsymbol{c}} \rightarrow \boldsymbol{\Sigma}_{c}^{*}+\bar{D}$ decay}

In this case, the decay is

$$
\left[P_{c}(4440)\left[\frac{1}{2} \frac{1-}{2}\right]_{0}\right] \rightarrow\left[\Sigma_{c}^{*}(--) 1 \frac{1-}{2}\right]+\left[\bar{D}(1870) \frac{1}{2} 0^{-}\right],
$$

with $l=1$ by parity. The formula remains the same as the preceding one, with the only change being the value of $n_{z}$ in the averaging over $\alpha$,

$$
\begin{aligned}
\Gamma_{P_{c}(J, S) \rightarrow \Sigma_{c}^{\star}+D}= & \frac{|\vec{p}|}{8 \pi M_{P_{c}}^{2}} \frac{\langle\alpha\rangle^{2}}{(2 J+1)}|\mathbb{A}|^{2} \times \sum_{s_{1}, s_{2}}\left|\left\langle S, s_{1}+s_{2} \mid \frac{1}{2}, s_{1} ; \frac{1}{2}, s_{2}\right\rangle\right|^{2}\left|\left\langle J, s_{1} \mid \frac{1}{2},-s_{2} ; S, s_{1}+s_{2}\right\rangle\right|^{2} \\
& \times\left|\left\langle 1,0 \mid \frac{1}{2}, s_{1} ; \frac{1}{2},-s_{1}\right\rangle\right|^{2}\left|\left\langle\frac{1}{2},-s_{1} \mid 1,0 ; \frac{1}{2},-s_{1}\right\rangle\right|^{2} \\
= & \frac{|\vec{p}|}{8 \pi M^{2}} \frac{\langle\alpha\rangle^{2}}{6(2 J+1)}|\mathbb{A}|^{2} \times \sum_{s_{1}, s_{2}}\left|\left\langle S, s_{1}+s_{2} \mid \frac{1}{2}, s_{1} ; \frac{1}{2}, s_{2}\right\rangle\right|^{2}\left|\left\langle J, s_{1} \mid \frac{1}{2},-s_{2} ; S, s_{1}+s_{2}\right\rangle\right|^{2} .
\end{aligned}
$$


However, since the incoming and outgoing states have different parity in the $z$ direction, the average of $\alpha$ will be zero in this case, hence

$$
\Gamma_{P_{c}(J, S) \rightarrow \Sigma_{c}^{\star}+D}=0 .
$$

\section{HIDDEN CHARM DECAY}

The $P_{c}(4440)$ state can strongly decay only through $J / \Psi$ with hidden charm because of kinematics,

$$
\left[P_{c}(4440)\left[\frac{1}{2} \frac{1-}{2}\right]_{0}\right] \rightarrow\left[J / \Psi(3097) 01^{-}\right]+\left[p(938) \frac{11^{+}}{2}{ }^{+}\right],
$$

with $l=0,2$. The decay momentum for the charm is about $|\vec{P}| \approx 809 \mathrm{MeV}$, so the final kinematics is relativistic. To determine the transition coupling $P_{c} \rightarrow J / \Psi+p$ we need the U(1) transition current

$$
\left\langle P_{c}\left[p_{2}, \frac{1}{2} \frac{1^{-}}{2}\right]\left|\rrbracket^{\mu}(0)\right| P\left[p_{1}, \frac{1}{2} \frac{1}{2}\right]\right\rangle,
$$

in which the in-out states in Eq. (61) are eigenstates of the moduli Hamiltonian defined earlier.

\section{A. Bulk-to-boundary current}

To determine Eq. (61), we consider the decay of pentaquarks into $J / \Psi$ (upsilon) represented by the $\mathrm{U}(1)$ vector field $\delta A_{\mu}(z) e^{-2 i m_{H} t}$ and the nucleon. To obtain the change in the Lagrangian one needs to select the terms that mixes the quark and antiquarks. One first considers $\delta A_{0}$. This leads to the temporal coupling

$$
\delta L_{T}=\delta A_{0} \frac{1}{m_{H}} \tilde{\rho}_{1},
$$

where

$\tilde{\rho}_{1}=\left(-\frac{9}{4 \tilde{\rho}^{2}} f^{2}+\frac{3}{16 \pi^{3} a} \frac{2 \rho^{2}-X^{2}}{\left(X^{2}+\rho^{2}\right)^{2}} f^{2}\right) \bar{u}_{Q} v_{\bar{Q}} \chi_{Q}^{\dagger} \chi_{\bar{Q}}^{\dagger}+$ H.c.

On the other hand, one also needs to consider $\delta A_{M}$, which contributes actually at leading order in $\lambda$, but next to leading order in $1 / m_{H}$. This amounts to a spatial coupling through

$$
\delta L_{S}=4 a N_{c} \lambda \delta A_{M} \partial_{N}\left(\Phi_{M}^{\dagger} \Phi_{N}-\Phi_{N}^{\dagger} \Phi_{M}\right),
$$

which is of order $1 / m_{H}$ in the heavy quark limit. $A_{M}$ sources a U(1) gauge field with bulk vector modes satisfying [21]

$$
-\left(1+Z^{2}\right)^{\frac{1}{3}} \partial_{Z}\left(\left(1+Z^{2}\right) \partial_{Z} \varphi_{n}(Z)\right)=\lambda_{n} \varphi_{n}
$$

and normalized according to

$$
\int d Z \frac{1}{\left(1+Z^{2}\right)^{\frac{1}{3}}}\left|\varphi_{n}(Z)\right|^{2}=1 .
$$

Recall that in the Sakai-Sugimoto model, the holographic coordinate $Z=z / U_{K K}$ with $U_{K K} \propto M_{K K}$. In the light-light sector, $M_{K K}$ is fixed to reproduce the low-lying rho-meson states $\tilde{m}_{n}=\lambda_{n} M_{K K}$ (odd $\left.n\right)$ [21].

In terms of the eigenmodes (65), the bulk-to-bulk vector propagator is given by

$G_{M N}\left(E ; \vec{P}, Z, X ; Z^{\prime}, X^{\prime}\right)=\frac{g_{M N}}{\tilde{\kappa}} \sum_{n} \frac{\varphi_{n}(Z) \varphi_{n}^{\dagger}\left(Z^{\prime}\right) e^{-i \vec{P} \cdot\left(\vec{X}-\vec{X}^{\prime}\right)}}{E^{2}-\vec{P}^{2}-\tilde{m}_{n}^{2}}$,

and the bulk-to-boundary $\mathrm{U}(1)$ gauge field is

$$
A^{M}(E ; Z, \vec{x}-\vec{X})=\frac{e^{-i \vec{P} \cdot(\vec{x}-\vec{X})}}{\sqrt{\tilde{\kappa}}} \sum_{n} \frac{\varphi_{n}(Z) a_{n}^{M}}{E^{2}-\vec{P}^{2}-\tilde{m}_{n}^{2}},
$$

for the spatial components $M=1,2,3, z$ with the bulk modular sources

$$
\begin{aligned}
& a_{n}^{Z}=\frac{4 \kappa}{8 m_{H} a N_{c}} i \vec{P} \cdot \bar{u}_{Q} \vec{\sigma} v_{\bar{Q}} \chi_{Q}^{\dagger} \chi_{\bar{Q}}^{\dagger}+\text { H.c., } \\
& \vec{a}_{n}=\frac{4 \kappa}{8 m_{H} a N_{c}} i \vec{P} \times \bar{u}_{Q} \vec{\sigma} v_{\bar{Q}} \chi_{Q}^{\dagger} \chi_{\bar{Q}}^{\dagger}+\text { H.c. },
\end{aligned}
$$

with $\chi_{Q, \bar{Q}}^{\dagger}$ fermionic creation operators in the pentaquark moduli satisfying anticommutation relations [11]. Here we have used the normalization condition $\int d Z d X f^{2}$ $\left(Z^{2}+X^{2}\right)=1$. Similarly, the bulk-to-boundary temporal component $A^{0}$ with full backreaction is

$$
A^{0}(E ; Z, \vec{x}-\vec{X})=\frac{e^{-i \vec{P} \cdot(\vec{x}-\vec{X})}}{\sqrt{\tilde{\kappa}}} \sum_{n} \frac{\varphi_{n}(Z) a_{n}^{0}}{E^{2}-\vec{P}^{2}-\tilde{m}_{n}^{2}},
$$

with the modular source

$$
\begin{aligned}
a_{n}^{0}= & \frac{4 \tilde{\kappa}}{a N_{c} m_{H}} \int d Z d^{3} X\left(-\frac{9}{4 \tilde{\rho}^{2}} f^{2}+\frac{3}{16 \pi^{3} a} \frac{2 \rho^{2}-X^{2}}{\left(X^{2}+\rho^{2}\right)^{2}} f^{2}\right) \\
& \times \bar{u}_{Q} v_{\bar{Q}} \chi_{Q}^{\dagger} \chi_{\bar{Q}}^{\dagger}+\text { H.c. }
\end{aligned}
$$

The boundary U(1) current sourced by the topological pentaquark in bulk follows from the canonical identification [21]

$$
\vec{\jmath}=-\left.\tilde{\kappa} \vec{F}^{z}\right|_{z=-\infty} ^{z=\infty}
$$

which is 


$$
\begin{aligned}
\vec{J}(\vec{x}-\vec{X})= & -\sum_{n} \frac{\lambda g_{n} \varphi_{n}(Z)}{4 m_{H} \sqrt{\tilde{\kappa}}} \int \frac{d^{3} \vec{P} e^{-i \vec{P} \cdot(\vec{x}-\vec{X})}}{E^{2}-\vec{P}^{2}-\tilde{m}_{n}^{2}} i \vec{P} \\
& \times \bar{u}_{Q} \vec{\sigma} v_{\bar{Q}} \chi_{Q}^{\dagger} \chi_{\bar{Q}}^{\dagger}+\text { H.c. }
\end{aligned}
$$

The pentaquark $\mathrm{U}(1)$ current at the boundary is sourced by the spin of the emerging $Q \bar{Q}$ attachment in bulk to order $1 / m_{H}$, with a $1^{--}$vector cloud composed essentially of the rho-meson Regge trajectory. This is not surprising given the holographic spin transmutation $\vec{J} \rightarrow \vec{J}+\vec{S}_{Q}$ discussed in [11]. This is the first major result in this section.

\section{B. Transition amplitude and width}

In terms of the boundary current (73), the transition form factor $P_{c} \rightarrow V+p$ reads

$$
\begin{aligned}
\left\langle P|\vec{\rrbracket}(\vec{x}-\vec{X})| P_{c}\right\rangle= & \left(i \vec{P} \times \bar{v}_{\bar{Q}} \vec{\sigma} u_{Q}\right) G(\vec{P}) \\
& \times(2 \pi)^{3} \delta^{3}\left(P^{\prime}-P\right),
\end{aligned}
$$

with the induced vector form factor

$$
G(\vec{P})=\lambda \sqrt{\frac{m_{N}}{M_{P_{c}}}} \sum_{n} \frac{\left\langle\varphi_{n}(Z)\right\rangle}{\sqrt{\tilde{\kappa}}} \frac{g_{n}}{E^{2}-\vec{P}^{2}-\tilde{m}_{n}^{2}} .
$$

The averaging in Eq. (75) is over the Gaussian baryonic (nucleon and pentaquark) modular wave functions which are localized around $Z \sim 0$ [11].

For comparison, we note that in the soft wall model, the bulk-to-boundary propagator $G(P, Z)$ can be expressed in terms of confluent hypergeometric functions $\mathcal{U}$ as

$$
\begin{aligned}
G(P, Z) \sim & M_{K K}^{2} Z^{2} \lambda \sqrt{\frac{m_{N}}{M_{P_{c}}}} \Gamma\left(1-\frac{P^{2}}{4 M_{K K}^{2}}\right) \mathcal{U} \\
& \times\left(1-\frac{-P^{2}}{4 M_{K K}^{2}} ; 2 ; M_{K K}^{2} Z^{2}\right) .
\end{aligned}
$$

The form factor follows by averaging over the Dirac fields in bulk. In contrast, the latter are localized around $Z \sim \infty$ to satisfy the hard scattering rules.

The scattering amplitude follows by LehmannSymanzik-Zimmermann reduction of Eq. (74),

$$
\mathcal{M}=\vec{\epsilon}^{\star} \cdot\left(i \vec{P} \times \bar{v}_{\bar{Q}} \vec{\sigma} u_{Q}\right) \lambda \sqrt{\frac{m_{N}}{M_{P_{c}}}} \frac{\left\langle\varphi_{n}(Z)\right\rangle}{\sqrt{\tilde{\kappa}}}
$$

for the emitted vector meson labeled by $V=n$. The squared scattering amplitude, after summing over the polarizations, reads

$$
\begin{aligned}
|\mathcal{M}|^{2}= & \frac{|\tilde{G}|^{2}|\vec{P}|^{2}}{2 S+1} \sum_{s_{1}, s_{2} ; s_{1}^{\prime} s_{2}^{\prime}}(\vec{n} \times \vec{\sigma})_{s_{1} s_{2}} \cdot(\vec{n} \times \vec{\sigma})_{s_{2}^{\prime} s_{1}^{\prime}} \\
& \times \sum_{M_{1}} C_{s_{1} s_{2}}^{S M_{1}} C_{s_{1}^{\prime} s_{2}^{\prime}}^{S M_{1}}
\end{aligned}
$$

with

$$
\tilde{G}(\vec{P})=\lambda \sqrt{\frac{m_{N}}{M_{P_{c}}}} \frac{\left\langle\varphi_{n}(Z)\right\rangle}{\sqrt{\tilde{\kappa}}},
$$

which can be reduced to

$$
\begin{aligned}
|\mathcal{M}|^{2} & =|\tilde{G}|^{2}|\vec{P}|^{2} \sum_{M_{1} ; s_{1} s_{2} ; s_{1}^{\prime} s_{2}^{\prime}} \frac{\vec{\sigma}_{s_{1} s_{2}} \cdot \vec{\sigma}_{s_{2}^{\prime} s_{1}^{\prime}}-\sigma_{s_{1} s_{2}}^{3} \sigma_{s_{1}^{\prime} s_{2}^{\prime}}^{3}}{2 S+1} C_{s_{1} s_{2}}^{S M_{1}} C_{s_{1}^{\prime} s_{2}^{\prime}}^{S M_{1}} \\
& =|\tilde{G}|^{2}|\vec{P}|^{2} \sum_{M_{1} ; s_{1} s_{2} ; s_{1}^{\prime} s_{2}^{\prime}} \frac{2 \delta_{s_{1} s_{1}^{\prime}} \delta_{s_{2} s_{2}^{\prime}}-\delta_{s_{1} s_{2}} \delta_{s_{1}^{\prime} s_{2}^{\prime}}-\sigma_{s_{1} s_{2}}^{3} \sigma_{s_{1}^{\prime} s_{2}^{\prime}}^{3}}{2 S+1} C_{s_{1} s_{2}}^{S M_{1}} C_{s_{1}^{\prime} s_{2}^{\prime}}^{S M_{1}} \\
& =\frac{2|\vec{P}|^{2}}{3}|\tilde{G}|^{2}\left(\delta_{S=1}+3 \delta_{S=0}\right) .
\end{aligned}
$$

Since the heavy quark in the initial state is still nonrelativistic, the decay rate for $P_{c} \rightarrow \gamma p$ is then

$$
\Gamma=|\vec{P}| \times \frac{|\vec{P}|^{2}}{4 \pi M_{P_{c}}^{2}} \frac{|\tilde{G}|^{2}}{2 S+1}
$$

or

$$
\Gamma=|\vec{P}| \times \frac{|\vec{P}|^{2}}{4 \pi(2 S+1) M_{P_{c}}^{2}} \frac{\lambda^{2} m_{N}}{M_{P_{c}}} \times\left|\frac{\left\langle\varphi_{n}(Z)\right\rangle}{\sqrt{\tilde{\kappa}}}\right|^{2} .
$$

The $J / \Psi$ bulk wave function satisfies a vector equation similar to Eq. (65) except for the overall scale. Indeed, recall that in the Sakai-Sugimoto construction $Z=z / U_{K K}$ and $U_{K K} \propto M_{K K}$, which is usually fixed by the light vector meson rho mass. For $J / \Psi$ we set $M_{K K} \rightarrow 2 m_{H}$ to the heavy meson mass, which parallels the construction in the soft wall model $[26,27]$. As a result, the bulk $J / \Psi$ wave functions follow from the bulk rho wave functions by rescaling

$$
\varphi_{n}(Z) \rightarrow \sqrt{\frac{M_{K K}}{2 m_{H}}} \varphi_{n}\left(\frac{M_{K K}}{2 m_{H}} Z\right),
$$


which leads to the partial decay width

$\Gamma=|\vec{P}| \times \frac{|\vec{P}|^{2}}{4 \pi(2 S+1) M_{P_{c}}^{2}} \frac{\lambda^{2} m_{N}}{M_{P_{c}}} \times \frac{M_{K K}}{M_{P_{c}}}\left(\frac{\varphi_{n}(0)}{\sqrt{\tilde{\kappa}}}\right)^{2}$

in the heavy quark limit.

We note the further suppression by $1 / m_{H}$ of the hidden decay width (84) in comparison to the open decay widths derived earlier. Indeed, a comparison with the open channel decay width yields the ratio

$\frac{\Gamma_{P_{c} \rightarrow J / \psi+P}}{\Gamma_{P_{c} \rightarrow \Lambda_{c}+\bar{D}}}=\lambda^{2}\left(\frac{16 \sqrt{2}}{2 S+1}\right)\left(\frac{|\vec{P}|^{3} m_{N} M_{K K}^{\frac{3}{2}}}{|\vec{p}| M_{P_{c}}^{\frac{9}{2}}}\right) \times\left(\frac{\left|\varphi_{n}(0)\right|^{2}}{\langle\alpha\rangle^{2}}\right)$.

The mismatch in the kinematical momenta in the ratio reflects the fact that the pentaquark decay to hidden charm follows from a Pauli-like coupling, while all open charm decays proceed from a Dirac-like coupling. For $P_{c}(440)$, the decay kinematics fixes $|\vec{P}| \approx 809 \mathrm{MeV}$ and $|\vec{p}| \approx 778 \mathrm{MeV}$. Using $M_{K K}=0.495 \mathrm{MeV}$ and $\lambda=g_{\mathrm{YM}}^{2} N_{c}=10$, the ratio (85) is

$$
\frac{\Gamma_{P_{c} \rightarrow J / \psi+P}}{\Gamma_{P_{c} \rightarrow \Lambda_{c}+\bar{D}}}=\frac{0.34\left|\varphi_{n}(0)\right|^{2}}{2 S+1} .
$$

The numerical value of the vector wave function at the origin solution to Eq. (65) is about $\frac{1}{2}$ for the ground state with $n=1$, so that

$$
\frac{\Gamma_{P_{c} \rightarrow J / \psi+P}}{\Gamma_{P_{c} \rightarrow \Lambda_{c}+\bar{D}}} \sim \frac{0.085}{2 S+1} .
$$

The decay width in the hidden channel is about $\frac{1}{10}$ the one observed in the open channels. This observation is in qualitative agreement with the one made using molecular bound states $[24,28]$.

For completeness and clarity, we have collected all the partial decay widths for charm pentaquark states, including their total width in units of $\Gamma$ [Eq. (40)] in Tables I-III. Overall, the decay widths of $P_{c}(4440)$ and $P_{c}(4312)$ are

TABLE I. Pentaquark $P_{c}(4440)\left[\frac{1}{2} \frac{1}{2}^{-}\right]_{0}$ decay widths in units of $\Gamma$.

\begin{tabular}{lcc}
\hline \hline Decay mode & Final momentum $(\mathrm{MeV})$ & Width \\
\hline$P_{c} \rightarrow \Lambda_{c} \bar{D}$ & $778 \mathrm{MeV}$ & 1 \\
$P_{c} \rightarrow \Sigma_{c} \bar{D}$ & $502 \mathrm{MeV}$ & 0.574 \\
$P_{c} \rightarrow \Lambda_{c} \bar{D}^{*}$ & $778 \mathrm{MeV}$ & 3 \\
$P_{c} \rightarrow \Sigma_{c}^{*} \bar{D}$ & $-\mathrm{MeV}$ & 0 \\
$P_{c} \rightarrow J / \Psi p$ & $809 \mathrm{MeV}$ & 0.085 \\
Total width & & 4.66 \\
\hline \hline
\end{tabular}

TABLE II. Pentaquark $P_{c}(4457)\left[\frac{1}{2} \frac{3}{2}\right]_{1}$ decay widths in units of $\Gamma$.

\begin{tabular}{lcc}
\hline \hline Decay mode & Final momentum $(\mathrm{MeV})$ & Width \\
\hline$P_{c} \rightarrow \Lambda_{c} \bar{D}$ & $801 \mathrm{MeV}$ & 0.68 \\
$P_{c} \rightarrow \Sigma_{c} \bar{D}$ & $537 \mathrm{MeV}$ & 0.409 \\
$P_{c} \rightarrow \Lambda_{c} \bar{D}^{*}$ & $801 \mathrm{MeV}$ & 2.04 \\
$P_{c} \rightarrow \Sigma_{c}^{*} \bar{D}$ & $-\mathrm{MeV}$ & 0 \\
$P_{c} \rightarrow J / \Psi p$ & $828 \mathrm{MeV}$ & 0.043 \\
Total width & & 3.172 \\
\hline \hline
\end{tabular}

TABLE III. Pentaquark $P_{c}(4312)\left[\frac{1}{2} \frac{1}{2}^{-}\right]_{1}$ decay widths in units of $\Gamma$.

\begin{tabular}{lcc}
\hline \hline Decay mode & Final momentum $(\mathrm{MeV})$ & Width \\
\hline$P_{c} \rightarrow \Lambda_{c} \bar{D}$ & $571 \mathrm{MeV}$ & 1.22 \\
$P_{c} \rightarrow \Sigma_{c} \bar{D}$ & $-\mathrm{MeV}$ & 0 \\
$P_{c} \rightarrow \Lambda_{c} \bar{D}^{*}$ & $571 \mathrm{MeV}$ & 3.66 \\
$P_{c} \rightarrow \Sigma_{c}^{*} \bar{D}$ & $-\mathrm{MeV}$ & 0 \\
$P_{c} \rightarrow J / \Psi p$ & $658 \mathrm{MeV}$ & 0.014 \\
Total width & & 4.894 \\
\hline \hline
\end{tabular}

found to be comparable, while the total decay width of $P_{c}(4457)$ is smaller. Within error bars, these observations are compatible with the charm pentaquark widths (1) reported by LHCb. To fix the value of $\Gamma$ in Eq. (40) (equivalently the value of the holographic parameter $\tilde{\kappa}$ ) and therefore all the remaining widths listed in the tables, we use the measured central value of the total width of $P_{c}(4440)$ in Eq. (1), namely

$$
\Gamma=\frac{20.6 \pm 4.9 \mathrm{MeV}}{4.66}=4 \pm 1 \mathrm{MeV}
$$

The yet to be observed bottom pentaquarks and their widths are listed in Table IV. For the bottom results, we used $m_{H}=5111 \mathrm{MeV}$ fixed by the heavy-light B-meson mass in Eq. (7). For the bottom pentaquark mass, we use the central holographic value $M_{P_{b}}=11163 \mathrm{MeV}$ [18], as the three predicted holographic bottom pentaquark masses are very close in mass. The differences in the widths listed

TABLE IV. Pentaquark $P_{b}(11163)\left[\left[\frac{1}{2} \frac{1}{2}^{-}\right]_{0} /\left[\frac{1}{2} \frac{1}{2}^{-}\right]_{1} /\left[\frac{1}{2} \frac{3}{2}^{-}\right]_{1}\right]$ decay widths in units of $\Gamma$.

\begin{tabular}{lcc}
\hline \hline Decay mode & Final momentum $(\mathrm{MeV})$ & Width \\
\hline$P_{b} \rightarrow \Lambda_{b} \bar{B}$ & $1206 \mathrm{MeV}$ & $2.38 / 3.96 / 1.58$ \\
$P_{b} \rightarrow \Sigma_{b} \bar{B}$ & $640 \mathrm{MeV}$ & $1.21 / 2.01 / 1.81$ \\
$P_{b} \rightarrow \Lambda_{b} \bar{B}^{*}$ & $1260 \mathrm{MeV}$ & $7.14 / 11.9 / 4.76$ \\
$P_{b} \rightarrow \Sigma_{b}^{*} \bar{B}$ & $-\mathrm{MeV}$ & 0 \\
$P_{b} \rightarrow \Upsilon_{p}$ & $1310 \mathrm{MeV}$ & $0.006 / 0.002 / 0.002$ \\
Total width & & $10.76 / 17.87 / 8.15$ \\
\hline \hline
\end{tabular}


stem from the different spin assignments. The broader width for bottom versus charm recorded in the tables

$$
\frac{\left[P_{b} \rightarrow \Lambda_{b} \bar{B}\right]}{\left[P_{c} \rightarrow \Lambda_{c} \bar{D}\right]} \sim 2.58
$$

stems from the larger momentum of the decay produce and the larger value for $m_{H}$. The much smaller ratio

$$
\frac{\left[P_{b} \rightarrow \Upsilon p\right]}{\left[P_{c} \rightarrow J / \Psi p\right]}=\left(\frac{M_{P_{c}}}{M_{P_{b}}}\right)^{\frac{9}{2}} \sim 0.02
$$

for fixed momentum decay follows from the larger suppression by the bottom pentaquark mass.

\section{CONCLUSIONS}

In leading order in the heavy quark mass $m_{H}$, the holographic construction predicts three heavy pentaquark states with the assignments $\left[\frac{1}{2} \frac{1}{2}^{-}\right]_{S=0,1}$ and $\left[\frac{1}{2} \frac{3}{2}^{-}\right]_{S=1}[11,12]$, which are BPS, degenerate and stable by heavy quark symmetry. In this limit, the heavy-light $\left[0^{-}, 1^{-}\right]=\left[D, D^{*}\right]$ multiplet binds democratically to an instanton core in bulk with equal spin and isospin. The core is stable by dual gauge-gravity interactions, and the ensuing dynamics has manifest chiral and heavy quark symmetries. The construction has very few parameters (three) with no need for ad hoc form factors.

The existence of three instead of two pentaquark states, as originally reported, is compatible with the recent reanalysis by the LHCb Collaboration [1], although the quantum number assignments are yet to be identified experimentally. The newly reported $P_{c}$ (4337) [17] state appears to be too low and narrow for an excited holographic pentaquark state $P^{*}$ candidate $[12,18]$. We also expect the chiral pentaquark doublers following from the addition of the mirror multiplet $\left[0^{+}, 1^{+}\right]=\left[\tilde{D}, \tilde{D}^{*}\right][29-31]$ to be more massive and even unbound.

We have shown how to systematically organize the spin corrections using the holographic bound state approach to the pentaquark states, away from the heavy quark mass limit. To order $1 / m_{H}$, spin effects lift the mass degeneracy through spin-orbit effects [18], and the pentaquark states undergo strong decays in channels with open and hidden charm (bottom). We have explicitly derived the spin induced vertices and used them to construct the pertinent transition amplitudes and form factors. Some of the transition form factors, e.g., $\gamma+p \rightarrow P_{c}$, may be accessible to precision photo- or electroexcitations of pentaquarks [32-34], as currently pursued at Jefferson Lab [35].

The transition couplings and form factors drive the strong decay widths of both charm and bottom pentaquarks, which are tied by symmetry to a single decay mode, say, $P_{c} \rightarrow \Lambda_{c}+\bar{D}$. In particular, the partial widths of the three pentaquark states are found to satisfy model independent ratios whenever allowed by kinematics. These observations carry to the bottom pentaquark states as well. The holographic analysis of the pentaquark states with hidden charm and bottom is extremely predictive and thus falsifiable.

\section{ACKNOWLEDGMENTS}

This work is supported by the Office of Science, U.S. Department of Energy, under Contract No. DE-FG88ER40388, and by Polish National Science Centre (NCN) Grant No. UMO-2017/27/B/ST2/01139.

\section{APPENDIX A: MODULI COEFFICIENT $\alpha$}

The moduli coefficient entering the Hamiltonian (23) follows from the averaging over the collective coordinates of the instanton

$$
\begin{aligned}
\alpha\left(\rho, \frac{Z}{\rho}\right) & =\frac{N_{c}}{2 \pi^{2} \sqrt{16 a N_{c}}} \int d \tilde{Z} d^{3} \tilde{X} \frac{c \phi_{n}(\tilde{Z}+Z)}{\left(\tilde{X}^{2}+\tilde{Z}^{2}+\rho^{2}\right)^{\frac{5}{2}}}\left(1+\frac{5 \rho^{2}}{2\left(\tilde{X}^{2}+\tilde{Z}^{2}+\rho^{2}\right)}\right) \\
& \rightarrow \frac{\rho N_{c}}{\sqrt{2} \pi^{3} \sqrt{16 a N_{c}}} \int d Z \phi_{n}(Z) \int d^{3} \tilde{X} \frac{1}{\left(\tilde{X}^{2}+Z^{2}+\rho^{2}\right)^{\frac{5}{2}}}\left(1+\frac{5 \rho^{2}}{2\left(\tilde{X}^{2}+Z^{2}+\rho^{2}\right)}\right) \\
& =\frac{\sqrt{2} \rho N_{c}}{3 \pi^{2} \sqrt{a N_{c}}\left(Z^{2}+\rho^{2}\right)} \int d Z \phi_{n}(Z),
\end{aligned}
$$

and depends on both the instanton size $\rho$ and the holographic $Z$ coordinate on the moduli. For our case we only need the modular wave function $n=1$, for which

$$
\int d Z \phi_{0}(Z)=2^{\frac{5}{8}} \pi^{\frac{1}{4}} \frac{1}{\sqrt{2 \kappa} m_{H}^{\frac{1}{4}} m_{K K}^{\frac{1}{4}}}
$$

and

$$
\left\langle\frac{\sqrt{2} \rho N_{c}}{3 \pi^{2} \sqrt{a N_{c}}\left(Z^{2}+\rho^{2}\right)}\right\rangle \sim \frac{4 \sqrt{2} N_{c}}{3 \pi}\left\langle\frac{\tilde{\rho}}{\tilde{\rho}^{2}+\tilde{Z}^{2}}\right\rangle .
$$

To carry the $\rho$-expectation value we need the radial wave functions for $l=1, l=0, l=2$ 


$$
R_{l=1,0,2}(\tilde{\rho})=\tilde{\rho}^{-1+\sqrt{(l+1)^{2}+\frac{36}{5}}} e^{-\frac{\tilde{\rho}^{2}}{\sqrt{6}}},
$$

and the modular wave function (non-normalized)

$$
\psi(Z)=e^{-\frac{Z^{2}}{\sqrt{6}}}
$$

The results for $k=0$ are

$$
\begin{aligned}
& \left\langle l=0\left|\frac{\tilde{\rho}}{\tilde{\rho}^{2}+\tilde{Z}^{2}}\right| l=1\right\rangle=0.35, \\
& \left\langle l=0\left|\frac{\tilde{\rho}}{\tilde{\rho}^{2}+\tilde{Z}^{2}}\right| l=1\right\rangle=0.43 .
\end{aligned}
$$

The ensuing numerical values associated with the transition coefficients in Eq. (23) are

$$
\begin{aligned}
& \langle\alpha\rangle_{l=1 \rightarrow l=0}=4 \sqrt{2} \times 0.35 \times 2^{\frac{5}{8}} \pi^{\frac{1}{4}} / \pi=4.08 / \pi, \\
& \langle\alpha\rangle_{l=1 \rightarrow l=2}=4 \sqrt{2} \times 0.43 \times 2^{\frac{5}{8}} \pi^{\frac{1}{4}} / \pi=4.97 / \pi .
\end{aligned}
$$

\section{APPENDIX B: PROPERTIES OF CLEBSCH-GORDAN COEFFICIENTS}

Here we detail our conventions for the Clebsch-Gordan coefficients used. We denote by $\left|j_{1} m_{1}\right\rangle$ and $\left|j_{2} m_{2}\right\rangle$ the state vector for the standard $2 j_{1}+1$ and $2 j_{2}+1$ irreducible representations of the $s u(2)$ Lie algebra. The tensor product splits into $J=\left|j_{1}-j_{2}\right|, \ldots\left|j_{1}+j_{2}\right|$ irreducible representations in the following way:

$$
|J M\rangle=\sum_{m_{1}, m_{2}}\left|j_{1} m_{1}\right\rangle\left|j_{2} m_{2}\right\rangle\left\langle j_{1} m_{1} ; j_{2} m_{2} \mid J M\right\rangle
$$

where $\left\langle j_{1} m_{1} ; j_{2} m_{2} \mid J M\right\rangle$ are the Clebsch-Gordan coefficients normalized according to

$$
\sum_{m_{1}, m_{2}}\left|\left\langle j_{1} m_{1} ; j_{2} m_{2} \mid J M\right\rangle\right|^{2}=1
$$

For simplicity we write

$$
\left\langle j_{1} m_{1} ; j_{2} m_{2} \mid J M\right\rangle \equiv C_{m_{1} m_{2}}^{J M} .
$$

To carry the sums in the text, we make use of the orthogonality relations, and the following symmetry properties:

$$
\begin{aligned}
& \left|\left\langle j_{1} m_{1} ; j_{2} m_{2} \mid J M\right\rangle\right|=\left|\left\langle j_{2} m_{2} ; j_{1} m_{1} \mid J M\right\rangle\right|, \\
& \left|\left\langle j_{1} m_{1} ; j_{2} m_{2} \mid J M\right\rangle\right| \\
& =\sqrt{\frac{2 J+1}{2 j_{1}+1}}\left|\left\langle J(-M) ; j_{2} m_{2} \mid j_{1}\left(-m_{1}\right)\right\rangle\right|,
\end{aligned}
$$

as well as the explicit relation

$$
\left|\left\langle j\left(M-\frac{s}{2}\right) ; \frac{1}{2} \frac{s}{2} \mid\left(j \pm \frac{1}{2}\right) M\right\rangle\right|=\sqrt{\frac{1}{2}\left(1 \pm \frac{s M}{j+\frac{1}{2}}\right)},
$$

if $j_{1}$ or $j_{2}$ is equal to $\frac{1}{2}$.
[1] R. Aaij et al. (LHCb Collaboration), Observation of a Narrow Pentaquark State, $P_{c}(4312)^{+}$, and of the Two-Peak Structure of the $P_{c}(4450)^{+}$, Phys. Rev. Lett. 122, 222001 (2019).

[2] R. Aaij et al. (LHCb Collaboration), Observation of $J / \psi p$ Resonances Consistent with Pentaquark States in $\Lambda_{b}^{0} \rightarrow$ $J / \psi K^{-} p$ Decays, Phys. Rev. Lett. 115, 072001 (2015).

[3] T. J. Burns, Phenomenology of $\mathrm{P}_{c}(4380)^{+}, \mathrm{P}_{c}(4450)^{+}$and related states, Eur. Phys. J. A 51, 152 (2015).

[4] J.-M. Richard, Exotic hadrons: Review and perspectives, Few-Body Syst. 57, 1185 (2016).

[5] R. F. Lebed, R. E. Mitchell, and E. S. Swanson, Heavyquark QCD exotica, Prog. Part. Nucl. Phys. 93, 143 (2017).

[6] A. Esposito, A. Pilloni, and A. D. Polosa, Multiquark resonances, Phys. Rep. 668, 1 (2017).

[7] S. L. Olsen, T. Skwarnicki, and D. Zieminska, Nonstandard heavy mesons and baryons: Experimental evidence, Rev. Mod. Phys. 90, 015003 (2018).
[8] F.-K. Guo, C. Hanhart, U.-G. Meißner, Q. Wang, Q. Zhao, and B.-S. Zou, Hadronic molecules, Rev. Mod. Phys. 90, 015004 (2018).

[9] M. Karliner, J. L. Rosner, and T. Skwarnicki, Multiquark states, Annu. Rev. Nucl. Part. Sci. 68, 17 (2018).

[10] M.-L. Du, V. Baru, F.-K. Guo, C. Hanhart, U.-G. Meißner, J. A. Oller, and Q. Wang, Revisiting the nature of the $P_{c}$ pentaquarks, J. High Energy Phys. 08 (2021) 157.

[11] Y. Liu and I. Zahed, Heavy baryons and their exotics from instantons in holographic QCD, Phys. Rev. D 95, 116012 (2017).

[12] Y. Liu and I. Zahed, Heavy and strange holographic baryons, Phys. Rev. D 96, 056027 (2017).

[13] M.-Z. Liu, Y.-W. Pan, F.-Z. Peng, M. S. Sánchez, L.-S. Geng, A. Hosaka, and M. P. Valderrama, Emergence of a Complete Heavy-Quark Spin Symmetry Multiplet: Seven Molecular Pentaquarks in Light of the Latest LHCb Analysis, Phys. Rev. Lett. 122, 242001 (2019). 
[14] C. W. Xiao, J. Nieves, and E. Oset, Heavy quark spin symmetric molecular states from $\bar{D}^{(*)} \Sigma_{c}^{(*)}$ and other coupled channels in the light of the recent LHCb pentaquarks, Phys. Rev. D 100, 014021 (2019).

[15] M.-L. Du, V. Baru, F.-K. Guo, C. Hanhart, U.-G. Meißner, J. A. Oller, and Q. Wang, Interpretation of the $\mathrm{LHCb} P_{c}$ States as Hadronic Molecules and Hints of a Narrow $P_{c}$ (4380), Phys. Rev. Lett. 124, 072001 (2020).

[16] M.-J. Yan, F.-Z. Peng, M. S. Sánchez, and M. P. Valderrama, Interpretations of the new LHCb $P_{c}(4337)^{+}$pentaquark state, arXiv:2108.05306.

[17] R. Aaij et al. (LHCb Collaboration), Evidence for a new structure in the $J / \psi p$ and $J / \psi \bar{p}$ systems in $B_{s}^{0} \rightarrow J / \psi p \bar{p}$ decays, arXiv:2108.04720.

[18] Y. Liu, M. A. Nowak, and I. Zahed, preceding paper, Holographic charm and bottom pentaquarks. I. Mass spectra with spin effects, Phys. Rev. D 104, 114021 (2021).

[19] S.-w. Li, Holographic heavy-baryons in the Witten-SakaiSugimoto model with the D0-D4 background, Phys. Rev. D 96, 106018 (2017).

[20] D. Fujii and A. Hosaka, Heavy baryons in holographic QCD with higher dimensional degrees of freedom, Phys. Rev. D 101, 126008 (2020).

[21] T. Sakai and S. Sugimoto, Low energy hadron physics in holographic QCD, Prog. Theor. Phys. 113, 843 (2005).

[22] Y. Liu and I. Zahed, Holographic heavy-light chiral effective action, Phys. Rev. D 95, 056022 (2017).

[23] M. I. Eides, V. Yu. Petrov, and M. V. Polyakov, Pentaquarks with hidden charm as hadroquarkonia, Eur. Phys. J. C 78, 36 (2018).

[24] Y.-H. Lin and B.-S. Zou, Strong decays of the latest LHCb pentaquark candidates in hadronic molecule pictures, Phys. Rev. D 100, 056005 (2019).

[25] K. Hashimoto, T. Sakai, and S. Sugimoto, Holographic baryons: Static properties and form factors from gauge/ string duality, Prog. Theor. Phys. 120, 1093 (2008).
[26] K. A. Mamo and I. Zahed, Diffractive photoproduction of $J / \psi$ and $\Upsilon$ using holographic QCD: Gravitational form factors and GPD of gluons in the proton, Phys. Rev. D 101, 086003 (2020).

[27] K. A. Mamo and I. Zahed, Electroproduction of heavy vector mesons using holographic QCD: From near threshold to high energy regimes, Phys. Rev. D 104, 066023 (2021).

[28] M. I. Eides and V. Yu. Petrov, Decays of pentaquarks in hadrocharmonium and molecular scenarios, Phys. Rev. D 98, 114037 (2018).

[29] M. A. Nowak, M. Rho, and I. Zahed, Chiral effective action with heavy quark symmetry, Phys. Rev. D 48, 4370 (1993).

[30] W. A. Bardeen, E. J. Eichten, and C. T. Hill, Chiral multiplets of heavy-light mesons, Phys. Rev. D 68, 054024 (2003).

[31] Y. Liu and I. Zahed, Heavy exotic molecules with charm and bottom, Phys. Lett. B 762, 362 (2016).

[32] Q. Wang, X.-H. Liu, and Q. Zhao, Photoproduction of hidden charm pentaquark states $P_{c}^{+}(4380)$ and $P_{c}^{+}(4450)$, Phys. Rev. D 92, 034022 (2015).

[33] V. Kubarovsky and M. B. Voloshin, Formation of hiddencharm pentaquarks in photon-nucleon collisions, Phys. Rev. D 92, 031502 (2015).

[34] M. Karliner and J. L. Rosner, Photoproduction of exotic baryon resonances, Phys. Lett. B 752, 329 (2016).

[35] Z.-E. Meziani and S. Joosten, Origin of the proton mass? Heavy quarkonium production at threshold from Jefferson Lab to an electron ion collider, in Proceedings of Probing Nucleons and Nuclei in High Energy Collisions: Dedicated to the Physics of the Electron Ion Collider, Seattle, WA, 2018 (World Scientific, 2020), 10.1142/9789811214950_ 0048.

[36] Y. Liu, K. A. Mamo, M. A. Nowak, and I. Zahed, following paper, Holographic charm and bottom pentaquarks. III. Excitations through photo-production of heavy mesons, Phys. Rev. D 104, 114023 (2021). 